\title{
Safety markers for rhabdomyosarcoma cells using an in vivo imaging system
}

\author{
SHUTONG DU ${ }^{1,3}$, LIAN MENG ${ }^{1}$, LINGXIE SONG ${ }^{1}$, PENGPENG ZHANG ${ }^{1}$, \\ XI SHOU ${ }^{4}$, CHUNXIA LIU ${ }^{1}$ and FENG LI ${ }^{1,2}$ \\ ${ }^{1}$ Department of Pathology, School of Medicine, Shihezi University, Shihezi, Xinjiang 832002; ${ }^{2}$ Department of Pathology, \\ Beijing Chaoyang Hospital, Capital Medical University, Beijing 100020; ${ }^{3}$ Department of Nursing, School of Medicine, \\ Xijing University, Xian, Shanxi 710123; ${ }^{4}$ Department of Animal Experiment Center, Xinjiang Key Laboratory for \\ Medical Animal Model Research, The First Affiliated Hospital of Xinjiang Medical University, \\ Ürümqi, Xinjiang 830011, P.R. China
}

Received February 13, 2017; Accepted March 7, 2018

DOI: $10.3892 / \mathrm{ol} .2018 .8789$

\begin{abstract}
In vivo imaging system (IVIS) is a novel and rapidly expanding technology that is widely applied in life sciences, including cell tracing. IVIS is able to quantify biological events, including tumor proliferation, through counting the number of photons emitted from a specimen. PLA802-enhanced green fluorescent protein (EGFP), PLA802-monomeric cherry fluorescent protein (mCherry), RH30-EGFP and RH30-mCherry tumor cells were injected into $18 \mathrm{BALB} / \mathrm{c}$ female nude mice subcutaneously with $5 \times 10^{6}$ cells in $100 \mu 1$ to quantitatively analyze EGFP and mCherry cells traced by IVIS. Inversion fluorescence microscopy revealed no transfection efficiency difference between PLA802-EGFP $(95.3 \pm 1.2 \%)$ and PLA802-mCherry $(95.8 \pm 1.7 \%)$, or between RH30-EGFP $(94.7 \pm 2.1 \%)$ and RH30-mCherry $(95.2 \pm 1.9 \%)$. Transfection did not influence the cell morphology of PLA802 or RH30. The cell migration, invasion and proliferation assay results of lentivirus-EGFP and lentivirus-mCherry revealed no significant difference prior to or following transfection. Therefore, lentivirus-EGFP and lentivirus-mCherry may serve as safety biological markers for PLA802 and RH30 cells. In vivo experiments demonstrated that lentivirus-EGFP and lentivirus-mCherry tumor luminescence signals were observed in all mice by IVIS. Hematoxylin-eosin staining and immunohistochemistry indicated that PLA802-EGFP, PLA802-mCherry, RH30-EGFP and RH30-mCherry cell lines exhibited rhabdomyosarcoma (RMS) characteristics like the maternal cells. In summary, mCherry and green fluorescent protein in
\end{abstract}

Correspondence to: Dr Chunxia Liu or Dr Feng Li, Department of Pathology, School of Medicine, Shihezi University, 211 Beisi Road, Shihezi, Xinjiang 832002, P.R. China

E-mail: liuliu2239@sina.com

E-mail: lifeng7855@126.com

Key words: in vivo, imaging system, rhabdomyosarcoma, lentivirus-enhanced green fluorescent protein, lentivirus-mCherry human RMS PLA802 and RH30 cancer cells may be safely and stably expressed for a long time in vitro and in vivo.

\section{Introduction}

Rhabdomyosarcoma (RMS) is a mesenchymal malignancy composed of neoplastic primitive precursor cells that exhibit histological features of myogenic differentiation (1). The most recent classification scheme of the World Health Organization subdivides RMS into four subtypes: Embryonal RMS (ERMS), pleomorphic RMS, spindle cell/sclerosing RMS and alveolar RMS (ARMS). The two major subtypes, ERMS and ARMS, differ in their genetic mutations, histological features, onset age and prognosis (2). ERMS is commonly diagnosed in children less than 10 years of age and is characterized by the presence of rhabdomyoblasts, a type of cell that has eccentric nuclei and eosinophilic cytoplasm and is embedded in a myxoid stroma. ARMS is commonly diagnosed in children over 10 years of age and is distinguished histologically by clusters of small, round, blue cells under high magnification (3). Patients with ARMS generally have a poorer clinical outcome than patients with ERMS. Therefore, understanding these aggressive tumors at the molecular level and obtaining insight into the biological differences between these patients and those with improved clinical outcomes is important. ARMS RH30 cells express the PAX-FOXO fusion protein, while PLA802 cells do not. Numerous studies on the RH30 cell line are available $(4,5)$, but those on the PLA802 cell line are few.

Fundamental research on tumors has been complicated by the lack of a technique that is able to label tumor cells for detected and tracking. The discovery and application of fluorescent protein has expanded the potential for tumor metastasis research. The commonly used fluorescent proteins are green fluorescent protein (GFP), red fluorescent protein (RFP), yellow fluorescent protein, and monomeric cherry fluorescent protein (mCherry). Fluorescent proteins exhibit stable expression, easy detection and high sensitivity, and are increasingly widely used in cell labeling and tracing. In cancer research, the fluorescent marker gene is able to display its position and quantity in the tumor cells and may provide 
a convenient condition for the study of the role of the gene in tumorigenesis and development, as well as the molecular mechanisms underpinning this (6-9).

Lentiviral vectors based on the human immunodeficiency virus are one of the most common tools for gene transfer and are able to infect non-dividing cells (10). The vector may effectively integrate foreign genes into the host chromosome to achieve persistent expression, and it is highly safe and rarely results in the immune response of the gene (11). Lentiviral vector may effectively infect numerous types of cells, including neuronal cells, cardiomyocytes, tumor cells and endothelial cells, in order to achieve a good gene therapy effect in the treatment of diseases. As a result, the vector has broad application prospects (12).

In the present study, RH30 and PLA802 cell lines were transfected with enhanced GFP (EGFP) and monomeric cherry fluorescent protein (mCherry) using a lentiviral vector, respectively. The cell biological characteristic changes in vivo and in vitro were also investigated. Finally, a cell that stably expressed fluorescence in vivo and in vitro and was observed using a PerkinElmer in vivo imaging system (IVIS; PerkinElmer, Inc., Waltham, MA, USA) for a long time was selected in providing an advantageous means for fundamental research into RMS.

\section{Materials and methods}

Cell culture. The human RMS PLA802 cell line was obtained from the Type Culture Collection of the Chinese Academy of Sciences (Shanghai, China), while the RH30 cell line was obtained from Shanghai Fuxiang Biotechnology Co., Ltd. (Shanghai, China). PLA802 cells were cultured in RPMI-1640 medium (Gibco; Thermo Fisher Scientific, Inc., Waltham, MA, USA), containing 10\% fetal bovine serum (FBS; Gibco; Thermo Fisher Scientific, Inc.) at $37^{\circ} \mathrm{C}$ in a humidified $5 \% \mathrm{CO}_{2}$ atmosphere. RH30 cells were cultured in Dulbecco's modified Eagle's medium (DMEM; Gibco; Thermo Fisher Scientific, Inc.), containing $10 \% \mathrm{FBS}$ at $37^{\circ} \mathrm{C}$ in a humidified $5 \% \mathrm{CO}_{2}$ atmosphere. Following two passages, cells were used for viral infection.

PLA802-EGFP/mCherry and RH30-EGFP/mCherry construction. hU6-MCS-Ubiquitin-EGFP-IRES-puromycin (EGFP-puro) and U6-MSC-Ubiquitin-Cherry-IRESpuromycin (mCherry-puro) lentiviral vectors were obtained from Shanghai GeneChem Co., Ltd. (Shanghai, China). In the preliminary experiment, logarithmic growth phase PLA802 and RH30 cells were seeded onto 96-well culture plates. Following overnight growth, cell confluence of $30-50 \%$ of $\sim 1 \times 10^{4}$ cells/well was observed. Enhanced infection solution from a lentivirus transfection kit (REVG0002; Shanghai GeneChem Co., Ltd., Shanghai, China) was diluted 10-fold in accordance with the virus particles and according to the manufacturer's protocol. With regards to lentivirus transfection, in terms of multiplicity of infection (MOI), the recommended range values were divided into four groups: i) MOI of 100, ii) MOI of 10, iii) MOI of 1 and iv) control group. Each group had three duplications and each well had a final volume of $100 \mu 1$. The 96-well culture plates were cultured in an incubator $\left(37^{\circ} \mathrm{C}, 5 \% \mathrm{CO}_{2}\right)$. After $8 \mathrm{~h}$, the plates were washed twice with phosphate-buffered saline (PBS) and $100 \mu 1$ fresh RPMI-1640 medium or DMEM were added to each well, followed by further cultivation for $72 \mathrm{~h}$ and transfection. The best MOI values of PLA802 and RH30 EGFP-puro and mCherry-puro cells were determined.

The obtained logarithmic growth phase PLA802 and RH30 cells were seeded onto 6-well culture plates. Each well had a final medium volume of $2 \mathrm{ml}$. After $8 \mathrm{~h}$, cells were washed twice with PBS, and $1 \mathrm{ml}$ fresh RPMI-1640 medium and DMEM were added to each well, followed by further cultivation for 2.5 days. The presence of fluorescence was determined $48 \mathrm{~h}$ after transfection at magnification, $\mathrm{x} 200$.

PLA802 and RH30 EGFP/mCherry-expressing cells obtained by puromycin. After 2-3 days of transfection, GFP and mCherry fluorescent signals were observed through an inverted fluorescence microscope. The initial puromycin screening concentration of $500 \mathrm{ng} / \mathrm{ml}$ obtained experimentally was combined with complete medium. Puromycin was obtained from Sigma-Aldrich (Merck KGaA, Darmstadt, Germany). The screening liquid was changed every two days. When no more dying cells were observed under the microscope, the screening concentration was reduced to $250 \mathrm{ng} / \mathrm{ml}$ to maintain a selective pressure. Culture was then continued for a week, and transfection efficiency (\%) was calculated using the following formula: Fluorescence cell number/total cell number $\mathrm{x} 100$.

Cell migration assays. For migration assays, 10,000 cells were resuspended in serum-free RPMI-1640 for PLA802 cells and serum-free DMEM for RH30 cells and placed in the upper chamber of a 24-well migration chamber (BD Biosciences, Franklin Lakes, NJ, USA). The lower chamber contained complete medium and 20\% FBS (Gibco; Thermo Fisher Scientific, Inc.). After $24 \mathrm{~h}$, cell migration was calculated as the percentage of total cells that had migrated to the bottom chamber, as observed under an Olympus BX51 fluorescent inverted microscope (Olympus, Tokyo, Japan) at magnification, $\mathrm{x} 200$.

Cell invasion assays. For invasion assays, 10,000 cells were re-suspended in serum-free RPMI-1640 for PLA802 cells and serum-free DMEM for RH30 cells (Gibco; Thermo Fisher Scientific, Inc.) and placed in the upper chamber of a 24-well Matrigel $^{\mathrm{TM}}$ invasion chamber (BD Biosciences) coated with Matrigel. The lower chamber contained complete medium and $20 \%$ FBS. Cell invasion was calculated after $48 \mathrm{~h}$, as the percentage of total cells that had invaded the bottom chamber, as observed under an Olympus BX51 fluorescent inverted microscope (Olympus, Tokyo, Japan) at magnification, x200.

Cell proliferation assays. Cell proliferation was assessed using the Cell Counting kit-8 (CCK-8; Dojindo Molecular Technologies, Inc., Kumamoto, Japan). The infected and control cells were seeded onto a 96-well microplate at a density of $2 \times 10^{3}$ cells/well. The cells were cultured for 7 days. The 96-well microplate was collected every $24 \mathrm{~h}$ to add $10 \mu \mathrm{l}$ CCK-8 solution to each well and was incubated at $37^{\circ} \mathrm{C}$ for an additional $3 \mathrm{~h}$. Optical density was determined at a wavelength of $450 \mathrm{~nm}$. 
Tumor xenograft assay. A total of 18 4-week-old female BALB/c nude mice (Vital River Laboratories Co., Ltd., Beijing, China) weighing 15-20 $\mathrm{g}$ had free access to food and water. The mice were housed at a temperature of $20-26^{\circ} \mathrm{C}$, a relative humidity of $40-70 \%$ and light/dark cycle of 12/12 h. All mouse experiments were approved by the Medical Animal Model Research of Xinjiang Key Laboratory. PLA802-EGFP, PLA802-mCherry, RH30-EGFP and RH30-mCherry tumor cells $\left(5 \times 10^{6}\right.$ in $\left.100 \mu \mathrm{l}\right)$ were injected subcutaneously into the left armpit region of the 18 female BALB/c nude mice using a $1 \mathrm{ml}$ syringe and a size 25 needle. Each group had three mice and mice were checked regularly for palpable tumors. Tumor size was measured weekly using calipers. Measurements of the height $(\mathrm{H})$, width (W) and depth (D) were collected and converted into relative tumor volumes $\left(\mathrm{HxWxD}, \mathrm{mm}^{3}\right)$. The maximum tumor volume observed in the present study was $147.618 \pm 20.258 \mathrm{~mm}^{3}$. An injection was scored as non-tumorigenic when a palpable tumor did not form after 120 days. The animal was sacrificed when a tumor reached $1 \mathrm{~cm}$ in diameter, and the tumor was harvested for histological analysis under anesthesia with intraperitoneal injection of $30-40 \mathrm{mg} / \mathrm{kg}$ pentobarbital.

Histopathological examination and immunohistochemistry. The animals were all sacrificed with $\mathrm{CO}_{2}$ (with the flow rate of $\mathrm{CO}_{2}$ euthanasia displacing $\leq 30 \%$ of the chamber volume/min) on day $120(\mathrm{n}=18)$. Tumor specimens were fixed in $10 \%$ formaldehyde solution at room temperature for $24 \mathrm{~h}$, paraffin embedded, subjected to routine H\&E staining (hematoxylin for $10 \mathrm{~min}$, eosin for $5 \mathrm{~min}$ at room temperature) and observed for morphology using a light microscope. Tumor embedding and staining were conducted at the Department of Pathology, Shihezi University School of Medicine (Xinjiang, China). Antigen retrieval was performed at $60^{\circ} \mathrm{C}$ for $2 \mathrm{~h}$, then the samples were dewaxed in xylene for $10 \mathrm{~min}$ and replaced with fresh xylene for $10 \mathrm{~min}$. Samples were then dehydrated in anhydrous ethanol for $5 \mathrm{~min}$, and then replaced with fresh anhydrous ethanol for $5 \mathrm{~min}, 95 \%$ ethanol for $2 \mathrm{~min}$, $90 \%$ ethanol for $2 \mathrm{~min}, 80 \%$ ethanol, $2 \mathrm{~min}, 70 \%$ ethanol for $2 \mathrm{~min}$ and tap water for 2 min Immunohistochemistry was performed using the Bench Mark XT automated system (Ventana Medical Systems, Inc., Tucson, AZ, USA) on 4-mm-thick, antigen-retrieved sections. The sections were incubated for $4^{\circ} \mathrm{C}$ overnight with primary antibodies against the following: MyoD1 (1:50; cat. no. M3512), myogenin (1:50; cat. no. M3559) and desmin (1:300; cat. no. M0760; all Dako; Agilent Technologies, Inc., Santa Clara, CA, USA). Subsequently, the sections were incubated with the secondary antibody, horseradish peroxidase-conjugated rabbit/mouse (cat. no. K5007; 100-200 $\mu$ l per slide; EnVision Detection Systems Peroxidase/DAB, Agilent Technologies, Inc.), for $30 \mathrm{~min}$ at $37^{\circ} \mathrm{C}$. Analysis was performed with a computerized image Aglient analysis system comprised of a photomicroscope and a digital camera. The entire tumor section on a slide was captured by consecutive fields with magnification, x200 and no overlapping.

Statistical analysis. All experiments were performed in three replicates, and the results were expressed as the mean \pm standard deviation. One-way analysis of variance followed by the least significant difference post-hoc test, was used to determine statistical significance. Statistical analysis was performed using SPSS version 22.0 (IBM Corp., Armonk, NY, USA). $\mathrm{P}<0.05$ was considered to indicate a statistically significant difference.

\section{Results}

Effect of lentiviral vectors on the morphology and transfection efficiency of cells. PLA802 and RH30 cells were observed with GFP and mCherry expression $72 \mathrm{~h}$ after transfection. Five fields for GFP and mCherry fluorescence cell counts were randomly selected. Next, stable expression of GFP and mCherry into PLA802 and RH30 cells was observed for seven days and compared with control cells using puromycin screening. More than $80 \%$ of cells were observed with GFP and mCherry expression (Fig. 1A and B), suggesting a high rate of infection. No transfection efficiency difference was observed between PLA802-EGFP $(95.3 \pm 1.2 \%)$ and PLA802-mCherry $(95.8 \pm 1.7 \%)$, or between RH30-EGFP $(94.7 \pm 2.1 \%)$ and RH30-mCherry $(95.2 \pm 1.9 \%)$. The results demonstrated that transfection did not influence the morphology of PLA802 or RH30 cells (Fig. 1A and B).

Effect of lentiviral vectors on cell migration, invasion and proliferation. Compared with control cell migration rates (74.2 \pm 5.3$)$, the migration rates of PLA802-EGFP cells

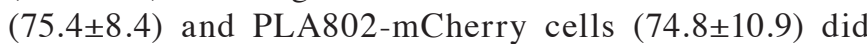
not exhibit a significant difference prior to and following transfection (Fig. 2A and B). Compared with control cell invasion rates $(88.4 \pm 10)$, the invasion rates of PLA802-EGFP cells $(86.6 \pm 9.1)$ and PLA802-mCherry cells $(89.8 \pm 5.9)$ did not exhibit a significant difference prior to and following transfection (Fig. 2A and B).

Compared with control cell migration rates $(73.7 \pm 20.1)$, the migration rate of RH30-EGFP cells $(72.7 \pm 16.6)$ and RH30-mCherry cells $(75.3 \pm 19.6)$ did not exhibit a significant difference prior to and following transfection (Fig. 2C and D). Compared with control cell invasion rates $(33.3 \pm 2.5)$, the migration rate of PLA802-EGFP cells (33.7 \pm 2.1$)$ and PLA802-mCherry cells (34.0 44.4$)$ did not exhibit a significant difference prior to and following transfection (Fig. 2C and D).

The proliferation rate of PLA802 and RH30 cells did not exhibit a significant difference prior to and following transfection (Fig. 2E and F).

Effect of lentiviral vector fluorescent protein expression in vivo. Following subcutaneous injection of PLA802-EGFP, PLA802-mCherry, RH30-EGFP and RH30-mCherry into $\mathrm{BALB} / \mathrm{c}$ female nude mice, subcutaneous tumors were observed in PLA802 cells and RH30 cells. The tumor formation rate was $100 \%$. Compared with the subcutaneous tumor growth rate, the volume of the tumor in the RH30 and PLA802 groups exhibited no significant difference between groups. The volume growth trend of each group was consistent (Fig. 3). Furthermore, fluorescence expression was observed in the two groups by IVIS (Fig. 3A).

Tumor histopathological examination and immunohistochemistry. H\&E staining revealed tumor cells 


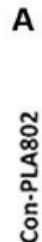
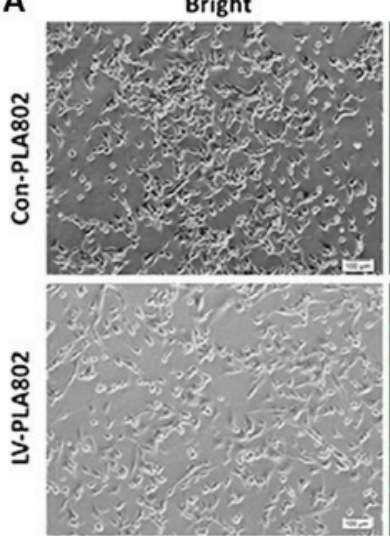

B
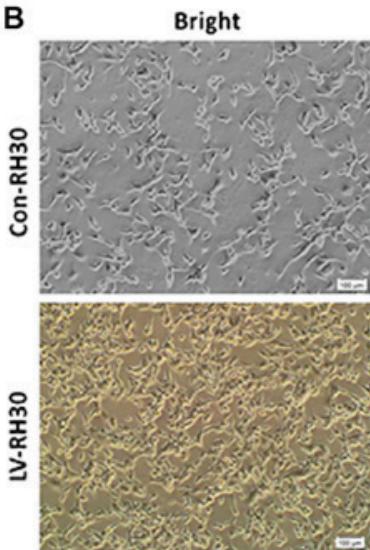
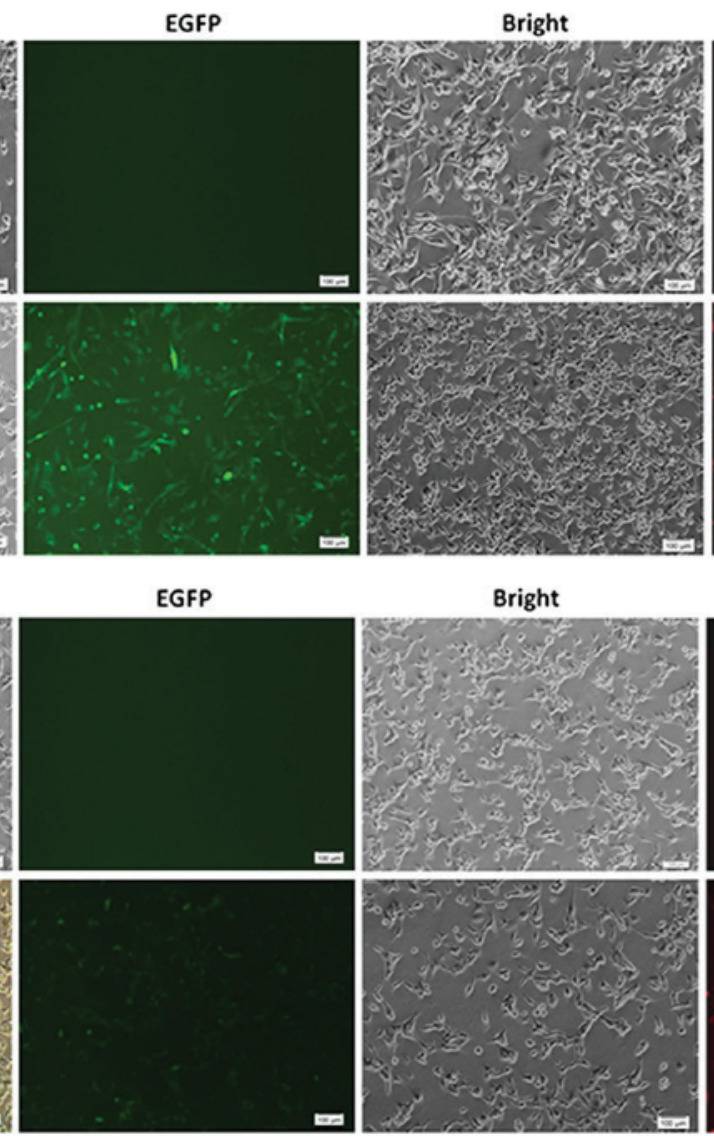
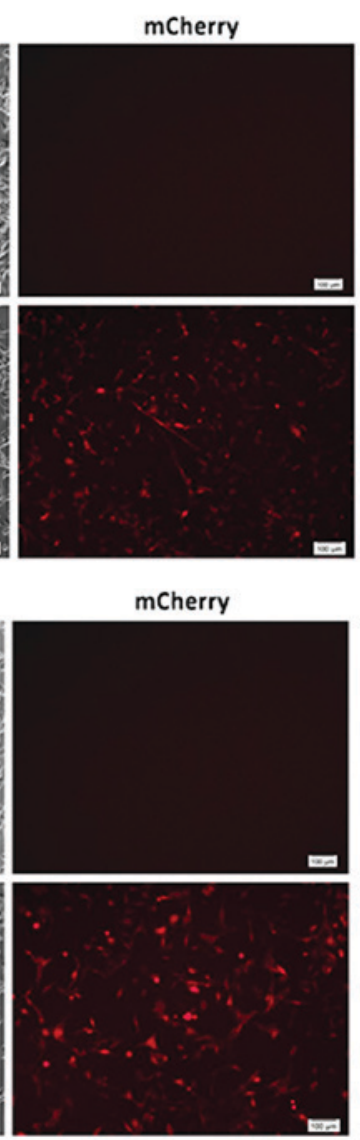

Figure 1. Identification of EGFP and mCherry protein expression in PLA802 and RH30 cells prior to and following transfection. (A) Intracellular fluorescence intensity and morphological changes of PLA802 and (B) RH30 cells. Scale bars: $100 \mu \mathrm{m}$. EGFP, enhanced green fluorescent protein; mCherry, monomeric cherry fluorescent protein; Con, control; LV, lentiviral vector.

with nest-like and alveolar arrangement, nuclei of round and oval shape, and a focal spot of a slight eosinophilic cytoplasm (Fig. 3C). Immunohistochemistry confirmed that tumors exhibited evidence of skeletal muscle differentiation. The tumors exhibited strong cytoplasmic desmin staining, as well as diffused nuclear MyoD1 and myogenin staining (Fig. 3C). They were composed of spindle-shaped cells with considerable atypical fibrillar material and lacked fibrovascular septa. Their histological phenotypes were similar to those of their corresponding original tumors.

\section{Discussion}

RMS is the most common type of soft tissue sarcoma in children (13). The prognosis for high risk patients with this disease has not improved significantly in the past few decades, and the survival rates of patients with metastatic or relapsed disease remain extremely poor (5). New clinical trials are slow and difficult, such that in vitro cell-line research and in vivo xenograft models present an attractive alternative for preclinical research for this tumor type (14). A total of 30 commonly used human RMS cell lines have been defined to date according to differing origins, karyotypes, histology and methods of validation. Selecting an appropriate cell line has important implications for the outcomes of RMS research (15). Therefore, studying RMS PLA802 and RH30 cells is important.
Given that RMS metastasis and recurrence occur from a single cell of the primary tumor, accurate tracking of tumor cell movement is particularly important. Understanding the tumor cells themselves may aid in the timely and accurate development of various interventions on the effect of the tumor (16). The study of malignant tumor metastasis and animal experiments has been stagnant for $\sim 10$ years, due to the lack of a transferable cell model. The development of optical imaging technology, including bioluminescence and fluorescence imaging, has made intuitive and accurate detection of tumor cell and gene behavior possible $(17,18)$. Bioluminescence imaging uses luciferase gene-labeled cells or DNA and has the advantage of strong specificity (19). In this technique, the substrate luciferin is injected into mice in the presence of ATP and oxygen. However, bioluminescence imaging has disadvantages, including instability, fast decay and toxicity (20). Fluorescence imaging uses fluorescent reporter gene labeling and is excited by excitation of fluorescent groups to achieve high energy; this technique emits light in a simple and intuitive manner. Fluorescence imaging is also able to mark various points and is therefore widely used in clinical and basic research $(21,22)$.

Weissleder et al (23) proposed molecular imaging in 1999 to understand the biological process at the cellular and molecular levels in vivo. Molecular imaging is a visualization of changes in cellular and molecular levels in vivo using imaging methods (24). Compared with in vitro detection, 
A

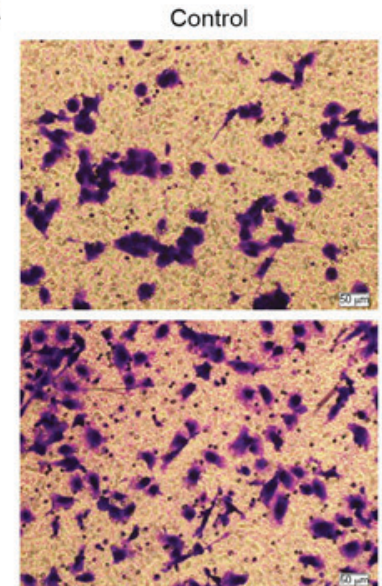

c

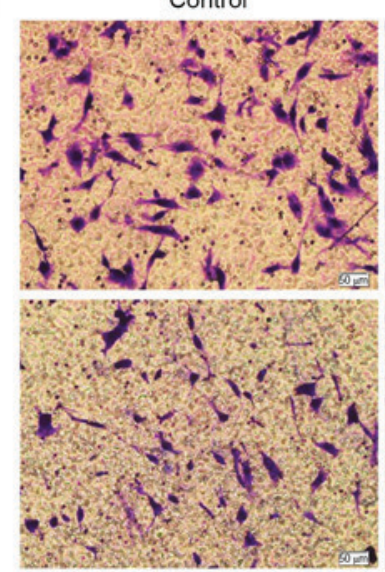

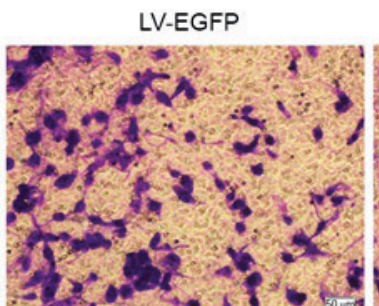

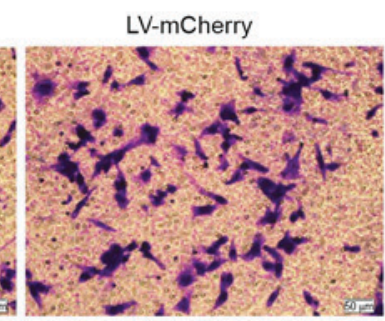

LV-mCherry

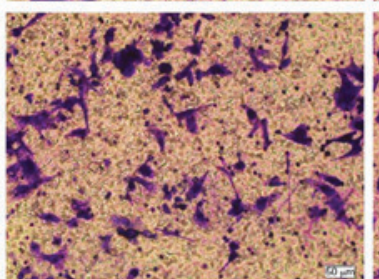

LV-EGFP
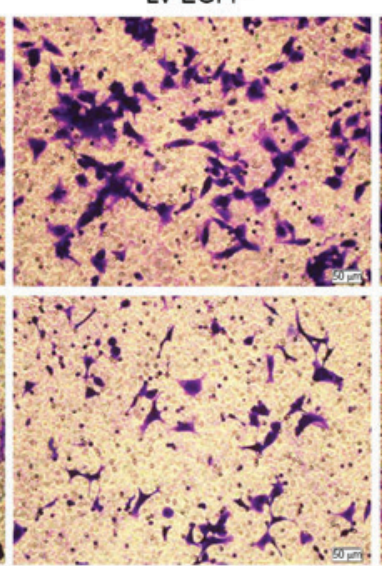

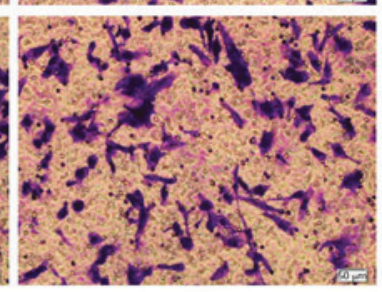

LV-mCherry
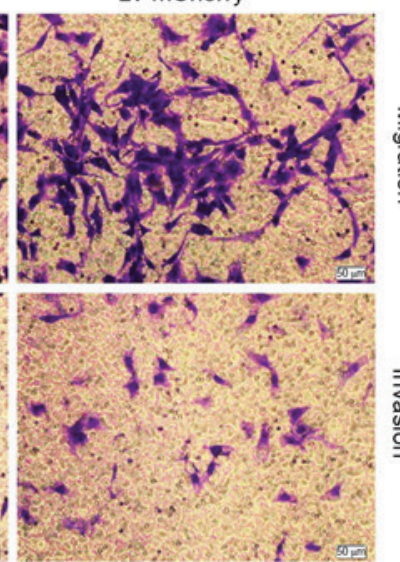

B
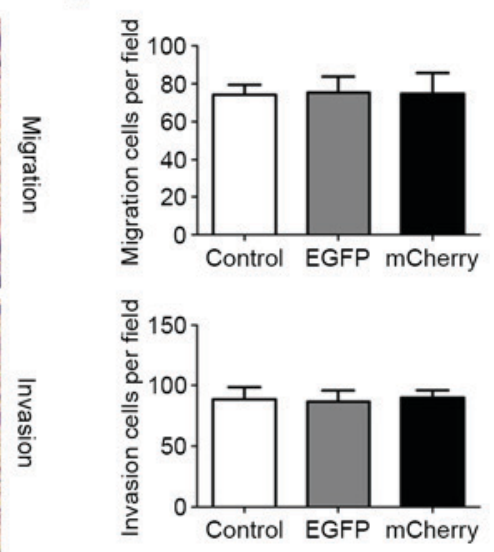

D
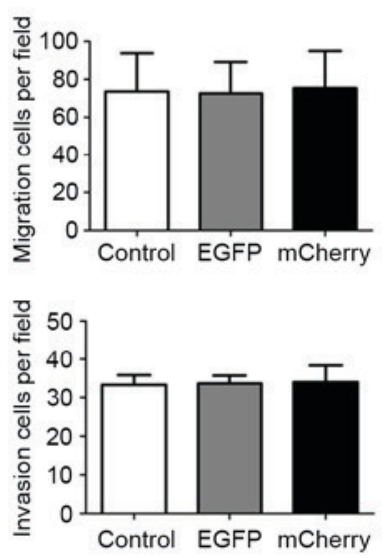

E
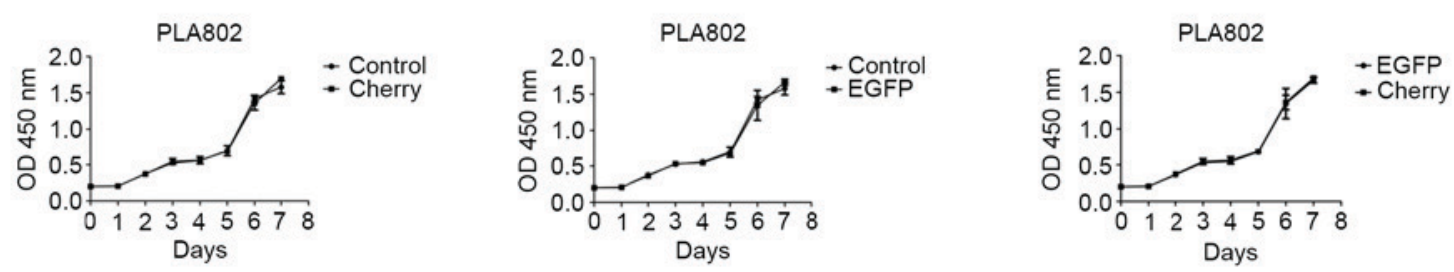

F
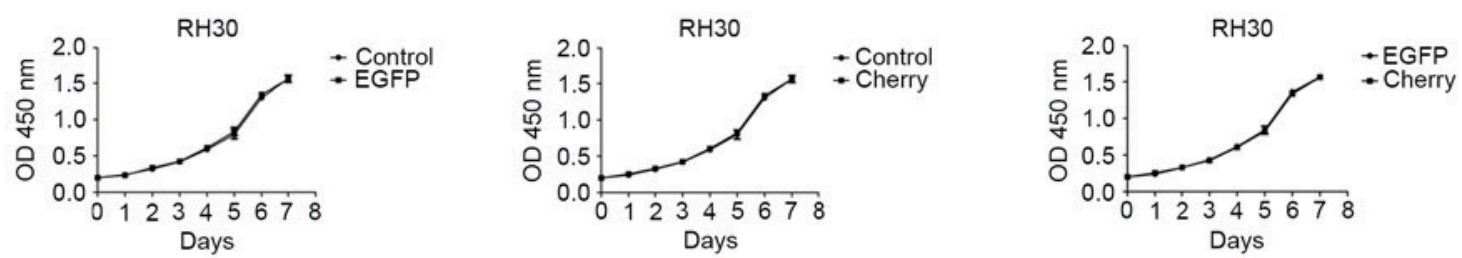

Figure 2. Cell function changes following lentivirus protein transfection. (A) Images and (B) statistical analyses of cell migration and invasion rates of PLA802 cells prior to and following transfection. (C) Images and (D) statistical analyses of cell migration and invasion rates of RH30 cells prior to and following transfection. (E) Proliferation rate of PLA802 cells for seven days prior to and following transfection. (F) Proliferation rate of RH30 cells for seven days prior to and following transfection. EGFP, enhanced green fluorescent protein; mCherry, monomeric cherry fluorescent protein; LV, lentiviral vector.

molecular imaging allows real-time identification, Compared with in vitro detection, molecular imaging allows real-time identification, molecular imaging can perform non-invasive dynamic observation of the human body and allows access to system information. This information focuses on changes in the level of genes and molecules, rather than the ultimate effect of gene molecular changes. The commonly used molecular imaging techniques for assessing gene expression in vivo include radionuclide imaging, magnetic resonance imaging and optical imaging (25). The development of imaging in the 21 st century has completely transformed from an era of anatomy or pathology imaging to a period of molecular imaging.

In vivo optical imaging primarily uses bioluminescence and fluorescence technologies. Bioluminescence imaging uses luciferase gene marker cells, while fluorescence imaging uses fluorescent genes to label and a very sensitive optical detection instrument to directly monitor the cell activity and gene behavior of the living organisms in vivo (26). The use of the characteristics of fluorescent proteins and the introduction of cells or transgenic animals in vivo may lead to non-invasive and dynamic longitudinal study of biological processes. 
A
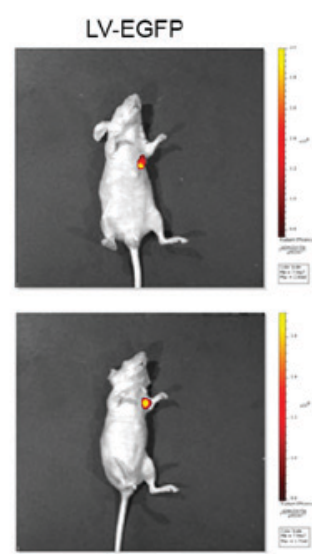

LV-mCherry
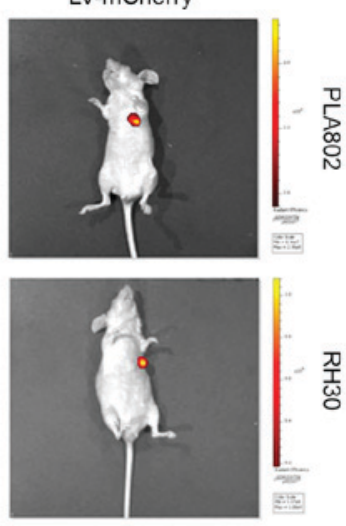
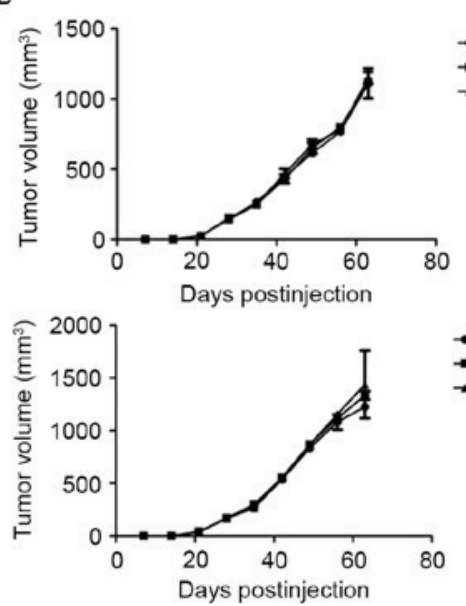

- PLA802

- PLA802-EGFP

- PLA802-mCherry

- $\mathrm{RH} 30$

- RH30-EGFP

- RH30-mCherry

C PLA802-EGFP

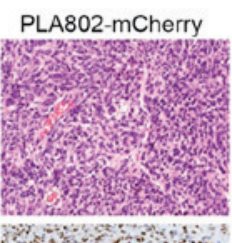
$\mathrm{RH} 30$

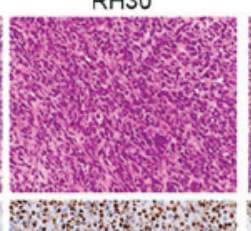

RH30-EGFP RH30-mCherry $H \& E$
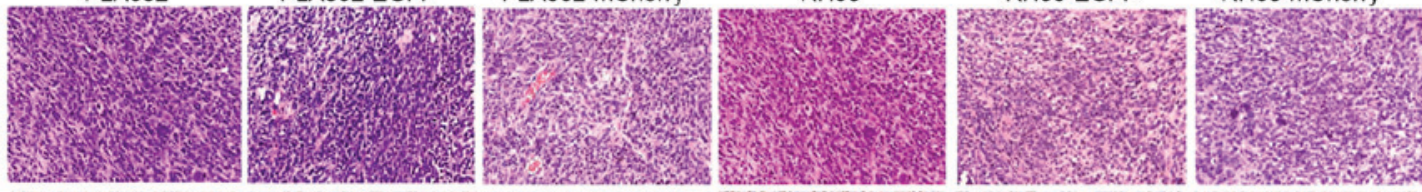

MyoD1
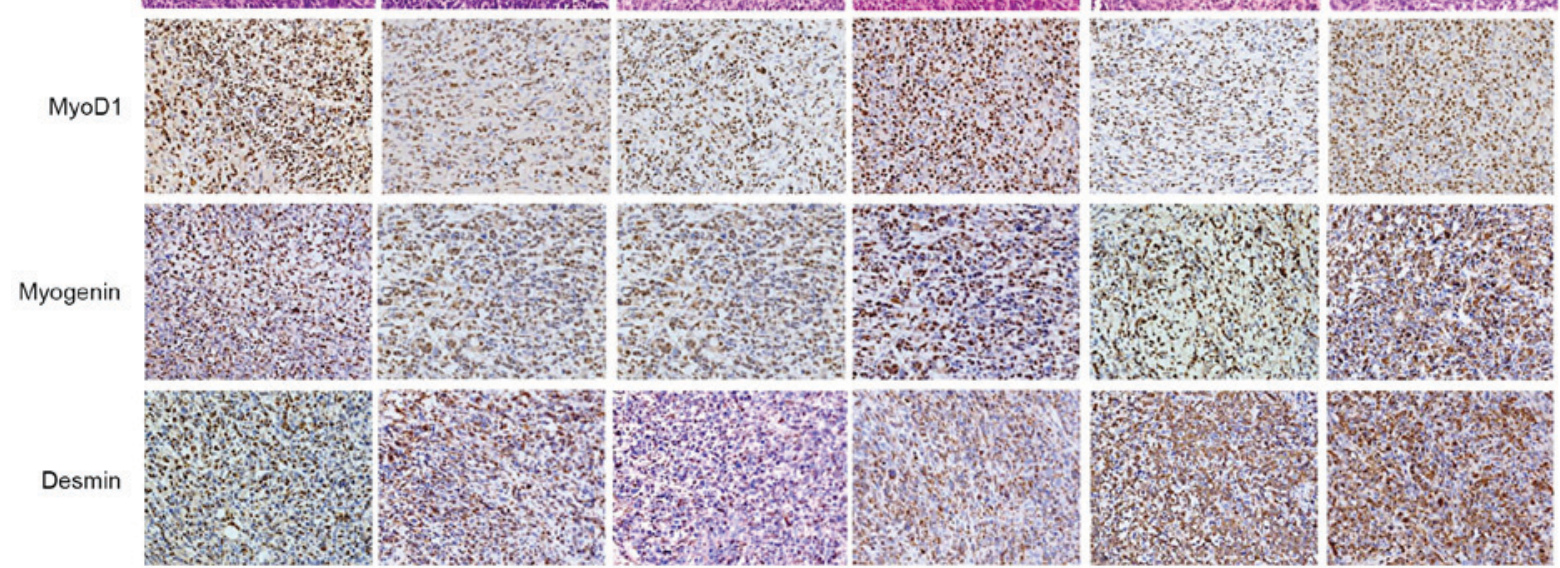

Figure 3. Stable rhabdomyosarcoma cells form tumors in vivo. (A) Monitoring of tumor growth dynamic by IVIS revealed EGFP and mCherry fluorescence signals in vivo. (B) Tumor volume $\left(\mathrm{mm}^{3}\right)$ was calculated from measurements of the height, width and depth of individual tumors. The tumor volume of EGFP and mCherry fluorescence groups exhibited no significant differences. (C) Tumors were analyzed by H\&E histopathology and immunohistochemical (magnification, x200) staining for desmin, MyoD1 and myogenin. EGFP, enhanced green fluorescent protein; mCherry, monomeric cherry fluorescent protein; $\mathrm{LV}$, lentiviral vector.

The discovery and application of GFP has provided a novel opportunity for tumor metastasis research. GFP was discovered by Osamu Shimomura in jellyfish, he found when jellyfish exposed range from the blue to the ultraviolet in light, it fluoresces green. In 1994, Chalfie et al (27) confirmed that GFP had compatibility with other living organisms. Since then, GFP has been used in the biological field. Adusumilli et al (28) introduced GFP to tumor cells and constructed a lymph node metastasis model. This model was used in the corresponding site of lymph node drainage and obtained accurate observation of lymph node micrometastasis and the range of the locations of its metastasis. Hoffman et al (22) established animal models of transplanted tumors using GFP-transfected tumor cells to determine the efficacy of various drugs. GFP applications have been improved, such as through the random and site-directed mutagenesis to obtain red-shifted fluorescent protein. Red-shifted fluorescence does not belong to the scope of autofluorescence and is conducive to reducing background autofluorescence (29).
RFP is a newly identified fluorescent protein gene isolated from an anemone in the Indian-Pacific Ocean. This gene emits red fluorescence under ultraviolet excitation with a maximum absorption wavelength of $558 \mathrm{~nm}$ and a maximum emission wavelength of $610 \mathrm{~nm}$ (30). RFP has a longer emission wavelength, higher sensitivity and a higher signal-to-noise ratio than GFP. This gene is a good complementary tool for the study of GFP in vivo. RFP is a natural luminescent protein with long excitation and emission wavelengths (31). The monomeric strawberry fluorescent protein (mStrawberry) and mCherry are mRFP1 chromophore residues and are direct mutations of the new fluorescent protein (mStrawberry and mCherry) (32). Given that the stability of mCherry is much stronger than that of mStrawberry, long-term imaging experiments on mRFP1 are the best alternative (33). In addition to $\mathrm{mKO}$ and TagRFP, these fruit-named fluorescent protein monomers also overcome the shortcomings of jellyfish red-shifted fluorescent proteins (such as YPet) and a large number of oligomeric red coral fluorescent proteins (34). 
Among various fluorescent protein labeling techniques, lentiviral transfection technology has the characteristics of broad spectrum, target gene transfection efficiency and high efficiency of chromosome integration (35). The technology improves the stability of fluorescent protein gene expression and avoids the in vitro selection process of fluorescence expressing cells, such that the chance of cell contamination is reduced (36). Lee et al (37) maintained in vivo stable expression of target cells for more than three months using retrovirus transfection of GFP. Long-term in vitro and in vivo observation revealed that the novel model retains the maternal biological properties while stably expressing the fluorescence.

In the present study, PLA802-EGFP, PLA802-mCherry, RH30-EGFP and RH30-mCherry cell lines were established by lentiviral transfection. After 180 days of in vitro culture, high expression of EGFP and mCherry fluorescence was observed. The aforementioned cell lines may stably express EGFP and mCherry, and may be passaged continuously to achieve long-term and stable labeling of cells. In vitro experiments, including cell invasion, migration and proliferation, revealed that the oncological characteristics of the cells were retained and consistent with the biological behavior of the maternal cells. The tumor growth rate was $100 \%$ and the growth of subcutaneous tumor was consistent. The proliferation activity of the two groups was similar to that of the maternal cells, but was different when under the influence of subcutaneous tumor formation. Tumor tissue blocks may display labeled fluorescence in small animal live imaging. The results of the present study revealed that the biological characteristics of the tumor cells were consistent with those of the maternal cells and did not change significantly. H\&E staining and immunohistochemistry revealed that PL802-EGFP, PLA802-mCherry, RH30-EGFP and RH30-mCherry cell lines exhibited RMS characteristics compared with maternal cells. The results demonstrated that tumor cell lines may provide a simple, direct and effective cancer cell line for revealing the invasion and metastasis of tumor cells. Therefore, these cells may be used to construct an RMS animal model, and the dynamic process of tumor cell growth and tumor formation may be observed stably, persistently, efficiently, intuitively and accurately by optical imaging.

In conclusion, an allograft model of RMS stably expressing EGFP and mCherry fluorescence in nude mice has the characteristics of a high tumorigenesis rate, strong repeatability, long-term observation, stable fluorescence characteristics, and stable and accurate expression in vivo. Different dual fluorescence labeling models may therefore be used to study circulating tumor cells. The model is suitable for the study of metastasis and recurrence of RMS, interventional therapy, imaging diagnosis and novel drug screening.

\section{Acknowledgements}

Not applicable.

\section{Funding}

The present study was supported by grants from the National Natural Science Foundation of China (grant nos. 81660441 and 81460404).

\section{Availability of data and materials}

The datasets used and analyzed during the current study are available from the corresponding author on reasonable request.

\section{Authors' contributions}

LM analyzed the cell migration data, LS analyzed the cell invasion data, PZ took the images from the Bench Mark XT automated system and analyzed the data, XS took the images from the IVIS and analyzed data. SD participated in all experimental projects and was a major contributor in writing the manuscript. CL and FL revised the manuscript for important intellectual content and analyzed the HE data.

\section{Ethics approval and consent to participate}

All mouse experiments were approved by the Medical Animal Model Research of Xinjiang Key Laboratory.

\section{Consent for publication}

Not applicable.

\section{Competing interests}

The authors declare that they have no competing interests.

\section{References}

1. Kashi VP, Hatley ME and Galindo RL: Probing for a deeper understanding of rhabdomyosarcoma: Insights from complementary model systems. Nat Rev Cancer 15: 426-439, 2015.

2. Parham DM and Ellison DA: Rhabdomyosarcomas in adults and children: An update. Arch Pathol Lab Med 130: 1454-1465, 2006.

3. Zhang M, Linardic CM and Kirsch DG: RAS and ROS in rhabdomyosarcoma. Cancer Cell 24: 689-691, 2013.

4. Erratum: Inhibition of rhabdomyosarcoma cell and tumor growth by targeting specificity protein $(\mathrm{sp})$ transcription factors. Int $\mathrm{J}$ Cancer 137: E9, 2015.

5. Hinson AR, Jones R, Crose LE, Belyea BC, Barr FG and Linardic CM: Human rhabdomyosarcoma cell lines for rhabdomyosarcoma research: Utility and pitfalls. Front Oncol 3: 183,2013

6. Ebe T: Green fluorescent protein as a marker gene expression. Tanpakushitsu Kakusan Koso 52(Suppl 13): 1766-1767, 2007 (In Japanese).

7. Zhang Q, Walawage SL, Tricoli DM, Dandekar AM and Leslie CA: A red fluorescent protein (DsRED) from discosoma $\mathrm{sp}$. as a reporter for gene expression in walnut somatic embryos. Plant Cell Rep 34: 861-869, 2015.

8. Katoh A, Shoguchi K, Matsuoka H, Yoshimura M, Ohkubo JI, Matsuura T, Maruyama T, Ishikura T, Aritomi T, Fujihara H, et al: Fluorescent visualisation of the hypothalamic oxytocin neurones activated by cholecystokinin-8 in rats expressing c-fos-enhanced green fluorescent protein and oxytocin-monomeric red fluorescent protein 1 fusion transgenes. J Neuroendocrinol 26: 341-347, 2014.

9. Fink D, Wohrer S, Pfeffer M, Tombe T, Ong CJ and Sorensen PH: Ubiquitous expression of the monomeric red fluorescent protein mCherry in transgenic mice. Genesis 48: 723-729, 2010.

10. Rossetti M, Cavarelli M, Gregori S and Scarlatti G: HIV-derived vectors for gene therapy targeting dendritic cells. Adv Exp Med Biol 762: 239-261, 2013 .

11. Chang LJ and He J: Retroviral vectors for gene therapy of AIDS and cancer. Curr Opin Mol Ther 3: 468-475, 2001.

12. Shichinohe T, Bochner BH, Mizutani K, Nishida M, Hegerich-Gilliam S, Naldini L and Kasahara N: Development of lentiviral vectors for antiangiogenic gene delivery. Cancer Gene Ther 8: 879-889, 2001. 
13. Dancsok AR, Asleh-Aburaya K and Nielsen TO: Advances in sarcoma diagnostics and treatment. Oncotarget 8: 7068-7093, 2017.

14. Shern JF, Yohe ME and Khan J: Pediatric rhabdomyosarcoma. Crit Rev Oncog 20: 227-243, 2015.

15. Casini N, Forte IM, Mastrogiovanni G, Pentimalli F, Angelucci A, Festuccia C, Tomei V, Ceccherini E, Di Marzo D, Schenone $\mathrm{S}$, et al: SRC family kinase (SFK) inhibition reduces rhabdomyosarcoma cell growth in vitro and in vivo and triggers p38 MAP kinase-mediated differentiation. Oncotarget 6: 12421-12435, 2015.

16. Liu C, Li D, Jiang J, Hu J, Zhang W, Chen Y, Cui X, Qi Y, Zou H, Zhang W and Li F: Analysis of molecular cytogenetic alteration in rhabdomyosarcoma by array comparative genomic hybridization. PloS One 9: e94924, 2014.

17. Ntziachristos V, Ripoll J, Wang LV and Weissleder R: Looking and listening to light: The evolution of whole-body photonic imaging. Nat Biotechnol 23: 313-320, 2005.

18. Maggi A and Ciana P: Reporter mice and drug discovery and development. Nat Rev Drug Discov 4: 249-255, 2005.

19. Dubey P: Reporter gene imaging of immune responses to cancer: Progress and Challenges. Theranostics 2: 355-362, 2012.

20. Martelli C, Dico AL, Diceglie C, Lucignani G and Ottobrini L: Optical imaging probes in oncology. Oncotarget 7: 48753-48787, 2016.

21. Hoffman RM: The multiple uses of fluorescent proteins to visualize cancer in vivo. Nat Rev Cancer 5: 796-806, 2005.

22. Hoffman RM: Fluorescent proteins as visible in vivo sensors. Prog Mol Biol Transl Sci 113: 389-402, 2013.

23. Weissleder R: Molecular imaging: Exploring the next frontier. Radiology 212: 609-614, 1999.

24. Chen ZY, Wang YX, Lin Y, Zhang JS, Yang F, Zhou QL and Liao YY: Advance of molecular imaging technology and targeted imaging agent in imaging and therapy. Biomed Res Int 2014: 819324, 2014.

25. Liu K, Wang MW, Lin WY, Phung DL, Girgis MD, Wu AM, Tomlinson JS and Shen CK: Molecular imaging probe development using microfluidics. Curr Org Synth 8: 473-487, 2011.

26. Hillman EM, Amoozegar CB, Wang T, McCaslin AF, Bouchard MB, Mansfield J and Levenson RM: In vivo optical imaging and dynamic contrast methods for biomedical research. Philos Trans A Math Phys Eng Sci 369: 4620-4643, 2011.
27. Chalfie M, Tu Y, Euskirchen G, Ward WW and Prasher DC: Green fluorescent protein as a marker for gene expression. Science 263: 802-805, 1994.

28. Adusumilli PS, Eisenberg DP, Stiles BM, Chung S, Chan MK, Rusch VW and Fong Y: Intraoperative localization of lymph node metastases with a replication-competent herpes simplex virus. J Thorac Cardiovasc Surg 132: 1179-1188, 2006.

29. Shen Y, Chen Y, Wu J, Shaner NC and Campbell RE: Engineering of mCherry variants with long Stokes shift, red-shifted fluorescence, and low cytotoxicity. PLoS One 12: e0171257, 2017.

30. Shen Y, Lai T and Campbell RE: Red fluorescent proteins (RFPs) and RFP-based biosensors for neuronal imaging applications. Neurophotonics 2: 031203, 2015.

31. Piatkevich KD and Verkhusha VV: Advances in engineering of fluorescent proteins and photoactivatable proteins with red emission. Curr Opin Chem Biol 14: 23-29, 2010.

32. Campbell RE, Tour O, Palmer AE, Steinbach PA, Baird GS, Zacharias DA and Tsien RY: A monomeric red fluorescent protein. Proc Natl Acad Sci USA 99: 7877-7882, 2002.

33. Merzlyak EM, Goedhart J, Shcherbo D, Bulina ME, Shcheglov AS, Fradkov AF, Gaintzeva A, Lukyanov KA, Lukyanov S, Gadella TW and Chudakov DM: Bright monomeric red fluorescent protein with an extended fluorescence lifetime. Nat Methods 4: 555-557, 2007.

34. Shaner NC, Campbell RE, Steinbach PA, Giepmans BN, Palmer AE and Tsien RY: Improved monomeric red, orange and yellow fluorescent proteins derived from discosoma sp. red fluorescent protein. Nat Biotechnol 22: 1567-1572, 2004.

35. Merten OW, Hebben M and Bovolenta C: Production of lentiviral vectors. Mol Ther Methods Clin Dev 3: 16017, 2016.

36. Rashidian M, Dozier JK and Distefano MD: Chemoenzymatic labeling of proteins: Techniques and approaches. Bioconjug Chem 24: 1277-1294, 2013.

37. Lee K, Majumdar MK, Buyaner D, Hendricks JK, Pittenger MF and Mosca JD: Human mesenchymal stem cells maintain transgene expression during expansion and differentiation. Mol Ther 3: 857-866, 2001. 